\title{
Evaluación preliminar del rendimiento energético y de la reducción de emisiones atmosféricas por la generación eléctrica del Sistema de Paneles Solares del Campus de la Universidad San Francisco de Quito en San Cristóbal - Galápagos - Ecuador
}

\author{
René Parra ${ }^{1 *}$ \\ ${ }^{1}$ Universidad San Francisco de Quito - Colegio de Ciencias e Ingeniería, Diego de Robles y Vía Interoceánica, Quito - Ecuador \\ Autor principal/Corresponding author, correo electrónico: rrparra@usfq.edu.ec
}

Editado por/Edited by: Cesar Zambrano, Ph.D.

Recibido/Received: 2015/03/11. Aceptado/Accepted: 2015/04/08.

Publicado en línea/Published online: 2015/05/22. Impreso/Printed: 2015/06/01.

\begin{abstract}
Renewable energy is an alternative to reduce the impacts from fossil fuel use. The control of atmospheric emissions is relevant in fragile ecosystems as the Galapagos Islands. At present almost all the electricity consumed in Galapagos is generated in power facilities equipped with internal combustion engines. This type of power facilities results in significant emissions of nitrogen oxides $\left(\mathrm{NO}_{x}\right)$, pollutants for which it has been established an annual average concentration of $30 \mu \mathrm{g} \mathrm{m}^{3}$ to protect sensible vegetation and ecosystems. In late July 2014, on the Campus of the Universidad San Francisco de Quito in Galapagos (USFQ Galapagos), a solar panels system began to operate. The preliminary assessment for the first four months of production indicates an average performance of $12.7 \%$. In one year this system avoids the emission of $11063 \mathrm{~kg}$ of $\mathrm{CO}_{2}$ and $298 \mathrm{~kg}$ of $\mathrm{NO}_{x}$, representing a $10.4 \%$ overall reduction of atmospheric emissions. Galápagos requires an air pollutant emission inventory and air quality monitoring, as basic tools for air quality management and control. This information will be useful to simulate the behavior of pollutants and understand the link between emissions, dispersion and the effects of air pollutants in the Galapagos Islands.
\end{abstract}

Keywords. internal combustion engine, single-crystalized panel, emission inventory, carbon footprint.

Preliminary evaluation of the energy efficiency and reduction of atmospheric emissions from the electricity generation system of solar panels on the campus of the Universidad San Francisco de Quito in San Cristobal Galapagos - Ecuador

Resumen

\begin{abstract}
Las energías renovables constituyen una alternativa para reducir los impactos del uso de combustibles fósiles. El control de las emisiones atmosféricas es relevante en ecosistemas frágiles como las Islas Galápagos. En la actualidad casi toda la electricidad que se consume en Galápagos se genera en centrales equipadas con motores de combustión interna. Estos equipos producen emisiones importantes de óxidos de nitrógeno $\left(\mathrm{NO}_{x}\right)$, contaminantes para los que se ha establecido una concentración media anual de $30 \mu \mathrm{g} \mathrm{m}^{3}$ a fin de proteger a la vegetación sensible y los ecosistemas. A finales de julio de 2014, en el Campus de la Universidad San Francisco en Galápagos (USFQ Galápagos), empezó a funcionar un Sistema de Paneles Solares (SPS). La evaluación preliminar de su funcionamiento durante los primeros 4 meses indica un rendimiento medio del 12.7\%. En un año el SPS evita la emisión de $11063 \mathrm{~kg}$ de $\mathrm{CO}_{2}$ y $298 \mathrm{~kg}$ de $\mathrm{NO}_{x}$, que representan una reducción global del $10.4 \%$ de las emisiones atmosféricas. Es prioritario contar con un inventario de emisiones atmosféricas y emprender campañas de monitoreo de la calidad del aire. Esta información permitirá también simular el comportamiento de los contaminantes. Estos componentes serán de gran utilidad para el control de la calidad del aire y de sus efectos en las Islas Galápagos.
\end{abstract}

Palabras Clave. motor de combustión interna, panel monocristalino, inventario de emisiones, huella de carbono.

\section{Introducción}

Las energías renovables constituyen una alternativa para reducir la dependencia y los impactos generados los combustibles fósiles, cuyo uso-entre otras connotacionesproduce emisiones de contaminantes atmosféricos y de gases de efecto invernadero. La reducción de estos impactos es prioritaria en regiones con ecosistemas frági- les, como las Islas Galápagos; donde el mayor porcentaje de electricidad para consumo público proviene de 4 generadoras equipadas con motores de combustión interna. Estas generadoras se localizan en las islas San Cristóbal, Santa Cruz, Isabela y Floreana (Fig. 1); funcionan con diésel y alimentan sistemas independientes, sin interconexión. Entre otros contaminantes del aire, este tipo de centrales emite cantidades importantes de 


\begin{tabular}{ccccccccc}
\hline \multicolumn{3}{c}{ Poder calorífico } & \multicolumn{3}{c}{ Factor de emisión de $\mathbf{C O}_{\mathbf{2}}$} & \multicolumn{3}{c}{ Densidad } \\
\hline Valor & Unidad & Fuente & Valor & Unidad & Fuente & Valor & Unidad & Fuente \\
\hline 43 & $\mathrm{TJ} \mathrm{Gg}^{-1}$ & {$[1]$} & 74100 & $\mathrm{~kg} \mathrm{TJ}^{-1}$ & {$[1]$} & 845 & $\mathrm{~kg} \mathrm{~m}^{-3}$ & {$[2]$} \\
\hline
\end{tabular}

Tabla 1: Propiedades del diésel.

óxidos de nitrógeno $\left(\mathrm{NO}_{x}\right)$, compuestos para los que se ha establecido una concentración media anual no mayor de $30 \mu \mathrm{g} \mathrm{m}^{3}$, con el fin de proteger la vegetación sensible y los ecosistemas [3, 4]. Los $\mathrm{NO}_{x}$ corresponden a la suma del óxido nítrico (NO) y del dióxido de nitrógeno $\left(\mathrm{NO}_{2}\right)$. Los resultados preliminares de estudios de la dispersión de los $\mathrm{NO}_{x}$ emitidos por estas generadoras, indican concentraciones medias de $\mathrm{NO}_{2}$ en 24 horas de hasta $35 \mu \mathrm{g} \mathrm{m}^{3}$ en el área de influencia directa de la generadora; y concentraciones entre 5 y 20 $\mu \mathrm{g} \mathrm{m}^{3}$ en la parte norte de la isla Santa Cruz [5]. Aun-
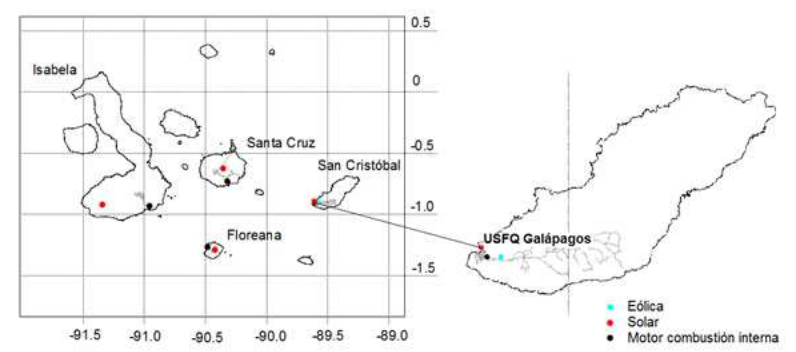

(a)

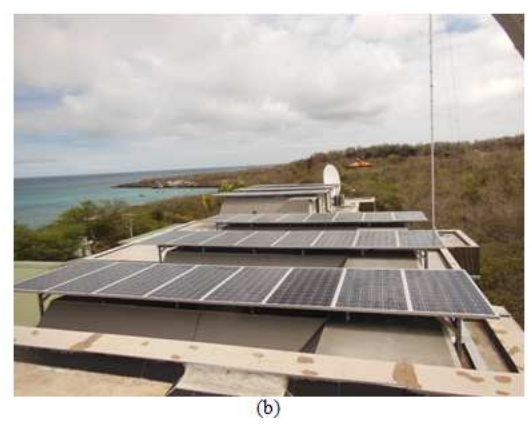

Figura 1: (a) infraestructura de generación eléctrica en las Islas Galápagos y localización del Campus de la Universidad San Francisco de Quito (USFQ Galápagos), (b) fotografía del SPS instalado en el edificio de la USFQ Galápagos (Autor: Sr. Luis Tasipanta).

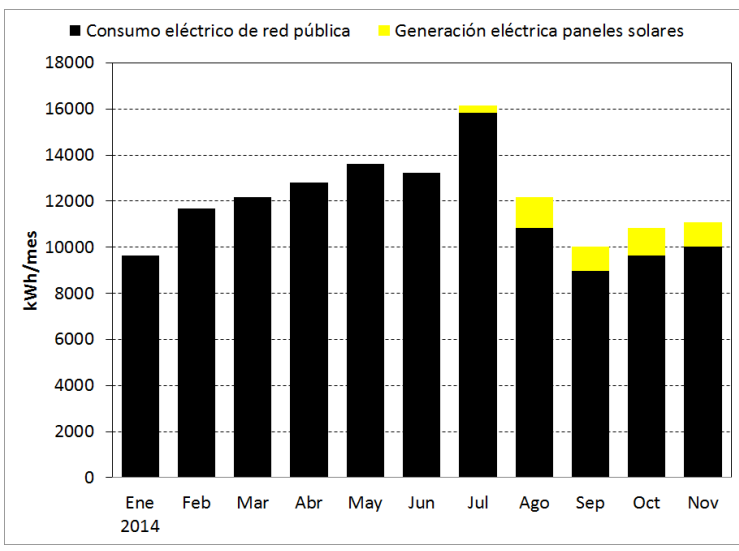

Figura 2: Consumo eléctrico en la USFQ Galápagos durante el periodo enero 2013 - noviembre 2014. EI SPS empezó a generar electricidad desde finales de julio de 2014. que estos resultados son preliminares, indican la necesidad de controlar y reducir las emisiones atmosféricas. La participación de fuentes renovables-como la eólica o fotovoltaica- es todavía marginal en Galápagos. Durante el año 2013, en San Cristóbal, el $99.7 \%$ de electricidad para consumo público (en total 7.87 GWh) fue suministrada por la generadora a diésel [6].

Por otra parte, la Universidad San Francisco de Quito inauguró en el año 2002 su extensión en San Cristóbal (USFQ Galápagos), con la misión de generar conocimiento, educar y formar a la Comunidad de las Islas Galápagos [7]. El consumo mensual de electricidad en la USFQ Galápagos, entre enero de 2013 y junio de 2014, varió entre 7.7 y 13.8 MWh (Fig. 2). La energía en este periodo fue adquirida en su totalidad a la Empresa Eléctrica Galápagos (EEG). A finales de julio de 2014 entró en funcionamiento un Sistema de Paneles Solares (SPS) que se instaló en el edificio de la USFQ Galápagos. Este sistema comprende 48 paneles monocristalinos, distribuidos en 3 grupos horizontales (Fig. 1), con un área total de $61.9 \mathrm{~m}^{2}\left(1.29 \mathrm{~m}^{2}\right.$ por panel). El sistema tiene una potencia total de $190 \mathrm{Wp}$.

Esta infraestructura reduce el consumo de electricidad generada con diésel, y por ello reduce también las emisiones atmosféricas de la USFQ Galápagos. En este artículo se presenta una evaluación del rendimiento energético durante los primeros 4 meses del funcionamiento del SPS y una estimación de las emisiones de contaminantes del aire y de dióxido de carbono $\left(\mathrm{CO}_{2}\right)$ que se han dejado de emitir.

\section{Método}

Se recolectaron los registros diarios de generación eléctrica del SPS, durante el periodo agosto - noviembre de 2014. Por otra parte, para el mismo periodo se procesaron los registros de la radiación solar global de la estación meteorológica instalada en la USFQ Galápagos. Con estos registros se obtuvo los valores diarios de

\begin{tabular}{lcc}
\hline & Categoría & $\begin{array}{c}\text { Factor de emisión } \\
\left(\mathbf{g ~ k W h} \mathbf{~ W h}^{-\mathbf{1}}\right)\end{array}$ \\
\hline $\mathrm{NO}_{x}$ & & 21.6 \\
$\mathrm{CO}$ & Contaminante & 4.7 \\
$\mathrm{COV}$ & primario & 1.8 \\
$\mathrm{SO}_{2}$ & & 2.3 \\
$\mathrm{MP}_{2,5}$ & & 0.5 \\
\hline $\mathrm{CO}_{2}$ & Gas de efecto & 802.6 \\
\hline
\end{tabular}

Tabla 2: Factores de emisión de contaminantes primarios y de $\mathrm{CO}_{2}$ por unidad de electricidad generada para consumo público en San Cristóbal - Galápagos. Año 2013. 


\begin{tabular}{|c|c|c|c|c|c|}
\hline & $\begin{array}{c}\text { Escenario 1: } \\
(\mathbf{k g}) \\
(\mathrm{A})\end{array}$ & $\begin{array}{c}\text { Escenario 2: } \\
\text { (kg) } \\
\text { (B) }\end{array}$ & $\begin{array}{c}\text { Emisiones } \\
\text { evitadas } \\
\text { Ago - Nov } 2014 \\
(\mathbf{k g}) \\
(\mathrm{B}-\mathrm{A})\end{array}$ & $\begin{array}{c}\text { Emisiones } \\
\text { evitadas } \\
\text { Ago - Nov } 2014 \\
(\%) \\
((\mathrm{B}-\mathbf{A}) / \mathrm{B}) * 100\end{array}$ & $\begin{array}{c}\text { Emisiones } \\
\text { evitadas } \\
\text { (kg) } \\
\text { Proyección } \\
\text { anual }\end{array}$ \\
\hline NOx & 853.3 & 952.5 & 99.3 & 10.4 & 297.8 \\
\hline $\mathrm{CO}$ & 183.6 & 205.0 & 21.4 & 10.4 & 64.1 \\
\hline $\mathrm{COV}$ & 69.6 & 77.7 & 8.1 & 10.4 & 24.3 \\
\hline $\mathrm{SO}_{2}$ & 92.0 & 102.7 & 10.7 & 10.4 & 32.1 \\
\hline $\mathrm{MP}_{2,5}$ & 19.8 & 22.1 & 2.3 & 10.4 & 6.9 \\
\hline $\mathrm{CO}_{2}$ & 31695.8 & 35383.5 & 3687.6 & 10.4 & 11062.9 \\
\hline
\end{tabular}

Escenario 1: Emisiones con la cantidad de electricidad adquirida a la EEA.

Emisiones bajo la asunción que el total de electricidad consumida (adquirida + la generada por el Sistema de Paneles Solares) fue suministrado por la EEG.

Tabla 3: Emisiones de contaminantes del aire y de $\mathrm{CO}_{2}$ evitadas por la generación eléctrica del Sistema de Paneles Solares de la USFQ Galápagos. Periodo agosto - noviembre de 2014.

la energía solar incidente. El rendimiento diario se estableció como la relación porcentual entre la generación eléctrica de los paneles y la energía solar incidente.

Para el cálculo de las emisiones de contaminantes primarios del aire y de $\mathrm{CO}_{2}$, se aplicó el modelo básico (Ec. 1) [8? ]:

$$
E_{j}=C o n E L \cdot F E_{j} / 1000
$$

Parámetro:

$j: \quad$ contaminante primario $j$, o de $\mathrm{CO}_{2}$.

Término:

$E_{j}$ : $\quad$ emisión del contaminante $j$, o de $\mathrm{CO}_{2}(\mathrm{t})$.

Datos:

ConEL: $\quad$ consumo de electricidad (kWh)

$F E_{j}$ : factor de emisión del contaminante $j$, o de $\mathrm{CO}_{2}\left(\mathrm{~g} \mathrm{kWh}^{-1}\right)$

Como contaminantes primarios se incluyeron a los $\mathrm{NO}_{x}$, monóxido de carbono (CO), compuestos orgánicos volátiles $(\mathrm{COV})$, dióxido de azufre $\left(\mathrm{SO}_{2}\right)$ y material particulado fino $\left(\mathrm{MP}_{2,5}\right)$.

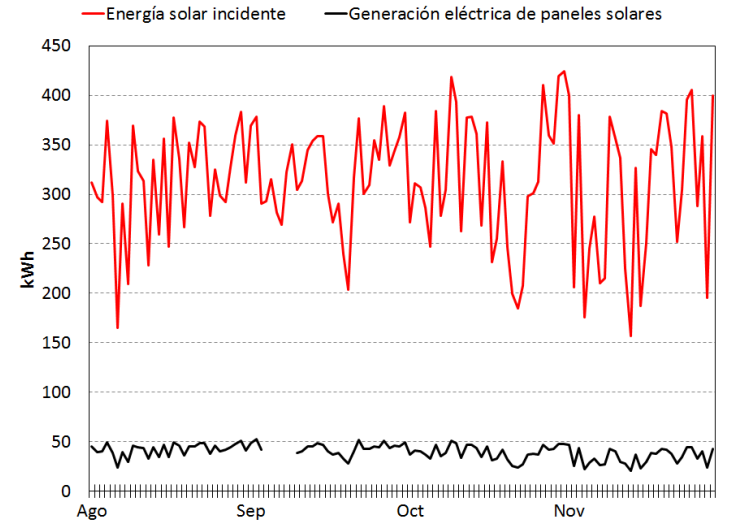

Figura 3: Energía solar incidente diaria y generación eléctrica diaria del Sistema de Paneles Solares de la USFQ Galápagos. Periodo agosto - noviembre de 2014.

\section{Factores de emisión}

El factor de emisión de $\mathrm{CO}_{2}$ se dedujo en base del consumo de combustible durante el año 2013 en la generadora de San Cristóbal (620 630 galones de diésel 2 [6]), y de las propiedades del diésel (Tabla 1). Con estos valores se calculó un total de $6.3 \mathrm{kt}$ de emisiones de $\mathrm{CO}_{2}$. La generación de electricidad para consumo público en San Cristóbal fue de 7.87 GWh [6]). Mediante la relación entre las emisiones y de generación eléctrica, se obtuvo un factor de emisión de $802.6 \mathrm{~g}$ de $\mathrm{CO}_{2}$ por $\mathrm{kWh}$. Se aplicó el mismo procedimiento para la deducción de los factores de emisión de contaminantes primarios, a partir de los factores de emisión AP 42 de la USEPA [9] para la generación de electricidad con diésel (distillate oil) en motores de combustión interna. El factor de emisión de dióxido de azufre $\left(\mathrm{SO}_{2}\right)$ corresponde a un contenido de azufre de $0.42 \%$. La Tabla 2 indica los factores de emisión obtenidos.

\section{Escenarios de emisión}

Se estimaron las emisiones atmosféricas correspondientes al consumo eléctrico en la USFQ Galápagos para dos escenarios: 1) con la cantidad de electricidad efectivamente adquirida a la EEG, y 2) bajo la asunción que el total de electricidad (adquirida a la EEG + la generada

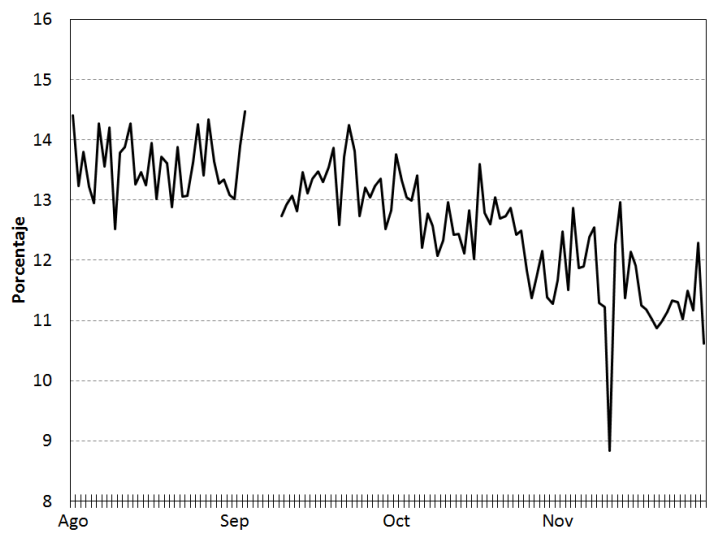

Figura 4: Rendimiento del Sistema de Paneles Solares de la USFQ Galápagos. Periodo agosto - noviembre de 2014. 
por el SPS) fue suministrado por la EEG. La diferencia de resultados de los dos escenarios corresponde a la reducción de emisiones por el aporte eléctrico del Sistema de Paneles Solares.

\section{Resultados y Discusión}

La energía solar incidente diaria varió entre 157.0 y 424.6 kWh (Fig. 3), con un valor medio de $312.6 \mathrm{kWh}$. La generación eléctrica diaria de los paneles solares varió entre 20.4 y $52.6 \mathrm{kWh}$ (Fig. 3), con un valor medio de 39.6 $\mathrm{kWh}$. El rendimiento energético diario, definido como la relación entre la electricidad generada sobre la energía solar incidente; varió entre 8.8 y $14.5 \%$ (Fig. 3), con un valor medio de $12.7 \%$. El rendimiento fue mayor durante agosto y septiembre, con un descenso paulatino en octubre y noviembre.

Los paneles solares comerciales presentan un rendimiento típico del $10 \%$ [10]. La Agencia Internacional de la Energía (AIE) indica que el rendimiento para paneles monocristalinos de uso comercial, varía entre 14 y $20 \%$ [11]. El rendimiento medio del SPS de la USFQ Galápagos se acerca al valor bajo del rango que reporta la AIE. En un $7 \%$ del periodo agosto - noviembre de 2014, el rendimiento diario varió entre 14 y $14.5 \%$, que comparable al valor inferior del rango indicado.

El rendimiento de los paneles solares depende de varios factores como los niveles de la radiación solar y su variación, la temperatura e inclinación de los paneles, las condiciones de limpieza, la resistencia de cables y dispositivos [12]. Se ha comprobado que la eficiencia se incrementa con la radiación solar, aunque baja con el aumento de la temperatura de los paneles [13, 14]. Se recomienda a futuro desarrollar una evaluación con al menos un año de registros, incluyendo un análisis de la influencia de la variación de la radiación solar, de la temperatura y de las condiciones de limpieza; con el fin de contar con un valor del rendimiento más representativo del SPS de la USFQ GAIAS. Su limpieza permanente promoverá un mayor rendimiento.

Durante los primeros 4 meses de funcionamiento, el SPS suministró el $11.5 \%$ de la electricidad consumida en la USFQ Galápagos. En un año evita la emisión de 11063 $\mathrm{kg}$ de $\mathrm{CO}_{2}$ y $298 \mathrm{~kg}$ de $\mathrm{NO}_{x}$ (Tabla 3), que representan un ahorro global del $10.4 \%$ de las emisiones atmosféricas.

Se recomienda desarrollar un diagnóstico del consumo energético con el fin de identificar potenciales acciones para optimizar la cantidad de electricidad que se adquiere a la EEG. Con ello se podría conseguir una reducción adicional de la huella de carbono y de las emisiones de contaminantes primarios del aire.

Es necesario contar con un inventario de emisiones de contaminantes del aire, tanto de fuentes naturales como antropogénicas, a fin de identificar y cuantificar el aporte de las fuentes de contaminación relevantes en Galápagos. Se requiere emprender campañas de monitoreo de la calidad del aire, para conocer los actuales niveles de los $\mathrm{NO}_{x}$ y de otros contaminantes. Esta información permitirá posteriormente simular el comportamiento de los contaminantes atmosféricos, y entender de mejor manera la interacción de los procesos de emisión, dispersión y efectos tanto en la población, la vegetación y el ecosistema de Galápagos.

\section{Agradecimientos}

Para el Sr. Luis Tasipanta, por su ayuda en la colecta de información.

\section{Referencias Bibliográficas}

[1] IPCC. 2006. "2006 IPCC Guidelines for National Greenhouse Gas Inventories, Prepared by the National. Greenhouse Gas Inventories Programme". Eggleston H.S., Buendia L., Miwa K., Ngara T. and Tanabe K. (eds).Published: IGES, Japan.

[2] USEPA. 1985. "Miscellaneous Data \& Conversion Factors". Appendix A.

[3] WHO. 2000. "Air Quality Guidelines for Europe. Second Edition". World Health Organization. Regional Office for Europe. Copenhagen.

[4] MfE. 2000. "Effects of Air Contaminants on Ecosystems and Recommended Critical Levels and Critical Loads. Final Report". Review of the Ambient Air Quality Guidelines. Air Quality Technical Report. New Zealand, (15).

[5] Parra, R. 2015. "Preliminary Assessment of the Chemical Transport of NOx Emissions from Power Facilities in the Galapagos Islands". World Energy Summit in the Galápagos, Ecuador. July 20-24.

[6] Conelec. 2015. "Estadística del Sector El'ectrico Ecuatoriano. Año 2013”. Quito - Ecuador.

[7] USFQ. 2015. "Universidad San Francisco de Quito". https://www.usfq.edu.ec/Paginas/Inicio.aspx.

[8] USEPA. 1995. "Compilation of Air Pollutant Emission Factors. Volume I: Stationary and Area Sources". AP42, Fifth Edition, U.S. Environmental Protection Agency. Research Triangle Park, NC 27711.

[9] USEPA. 2012. "Environmental Protection Agency". http://www.epa.gov/ttnchief1/ap42.

[10] Mackay, D. 2008. "Sustainable Energy - without the hot air". Cambridge - England.

[11] IEA. 2010. “Technology Roadmap. Solar Photovoltaic Energy". International Energy Agency. Paris - France.

[12] IEA. 2014. “Technology Roadmap. Solar Photovoltaic Energy". International Energy Agency. Paris - France.

[13] Melis, W.; Mallick, S.; Relf, P. 2014. "Increasing Solar Panel Efficiency in a Sustainable Manner". Conference paper. International Energy Conference - ENERGYCON. Dubrovnik. Croatia. 
[14] Yerli, B.; Kaymak, M.; Izgi, E.; Oztopal, A.; Sahin, A. 2010. "Effect of Derating Factors on Photovoltaics under Climate Conditions of Stambul". International Journal of Electrical, Computer, Electronics and Communication Engineering, 4(8):35-39. 